\title{
A retrospective cohort study assessing acute kidney injury and renal recovery among septic patients empirically treated with vancomycin piperacillin-tazobactam versus vancomycin cefepime
}

\author{
Brian Pacca Elliott ${ }^{1,2,3}$ - Michael M Tang ${ }^{1,2,3} \cdot$ Joshua Alexander Madden ${ }^{1,3} \cdot$ Ronald James Markert $^{3}$. \\ Steven Dale Burdette ${ }^{3,4}$. Craig Matthew Pleiman ${ }^{4,5}$. Emily Claire Speelmon ${ }^{1,3}$
}

Received: 12 March 2021 / Accepted: 21 May 2021 / Published online: 5 June 2021

(C) This is a U.S. government work and not under copyright protection in the U.S.; foreign copyright protection may apply 2021

\begin{abstract}
Vancomycin plus piperacillin-tazobactam (VPT) is a commonly used antimicrobial regimen for septic patients. VPT is more nephrotoxic than other regimens such as vancomycin plus cefepime (VC) when given over several days. This risk of nephrotoxicity is less clear when VPT is given for initial empiric therapy in sepsis and de-escalated quickly based on evolving clinical information. The objective of this study was to assess nephrotoxicity among septic patients empirically treated with either VPT or VC at initial clinical presentation. We conducted a retrospective study of septic patients who received VPT or VC within $12 \mathrm{~h}$ of presentation to the emergency department. The primary outcomes were acute kidney injury (AKI) and renal recovery $72 \mathrm{~h}$ after presentation. For the total of 418 patients, 306 received VPT and 112 received VC. Rates of $\mathrm{AKI}$ at $72 \mathrm{~h}$ were $15.2 \%$ for VPT patients and $11.0 \%$ for VC patients [p $=0.44]$. Among patients with AKI at presentation, $16.3 \%$ of VPT patients had AKI at $72 \mathrm{~h}$ compared to $8.9 \%$ of VC patients [ $p=0.19]$. Among those without AKI at presentation, $14.2 \%$ VPT patients and $16.7 \% \mathrm{VC}$ patients had AKI at $72 \mathrm{~h}[p=0.71]$. Renal recovery rates for patients with AKI at presentation were $42.3 \%$ for VPT patients versus $40.3 \%$ for VC patients [ $p=0.78$ ]. In-hospital renal replacement therapy occurred in $6.2 \%$ VPT patients and $0.9 \%$ VC patients [ $p=0.024]$. Therefore, initial empiric therapy with VPT in sepsis may not confer increased risk of AKI when de-escalated appropriately.
\end{abstract}

Keywords Sepsis · Acute kidney injury · Anti-bacterial agents $\cdot$ Critical care $\cdot$ Septic shock $\cdot$ Renal insufficiency

$\begin{array}{ll}\text { Abbreviations } \\ \text { AKI } & \text { Acute kidney injury } \\ \text { VPT } & \text { Vancomycin and piperacillin-tazobactam } \\ \text { VC } & \text { Vancomycin and cefepime } \\ \text { RRT } & \text { Renal replacement therapy }\end{array}$

Brian Pacca Elliott

Brian.Elliott@wright.edu

1 Department of Critical Care Medicine, Miami Valley Hospital, Dayton, OH, USA

2 Department of Internal Medicine, Wright Patterson Air Force Base, Dayton, OH, USA

3 Department of Internal Medicine and Neurology, Boonshoft School of Medicine, Wright State University, 128 E Apple St, Weber CHE Building, Dayton, OH 45409, USA

4 Department of Infectious Diseases, Miami Valley Hospital, Dayton, OH, USA

5 Department of Pharmacy, Miami Valley Hospital, Dayton, $\mathrm{OH}, \mathrm{USA}$
SOFA Sequential organ failure assessment

MAP Mean arterial pressure

\section{Introduction}

Sepsis accounts for approximately $6 \%$ of hospitalizations in the United States, with mortality rates of approximately $15-31 \%[1,2]$. Due to their severity of illness, septic patients require urgent and often broad-spectrum antibiotics to decrease mortality $[3,4]$. Prompt and appropriate antimicrobial therapy can also reduce the risk of sepsis-associated acute kidney injury (AKI), a frequent comorbidity of septic patients that occurs in $22-51 \%$ of patients with severe sepsis [5, 6]. However, nephrotoxic medications can also contribute to AKI in sepsis, accounting for approximately $20 \%$ of cases [6]. Therefore, an antimicrobial regimen that provides adequate broad-spectrum coverage but mitigates the risk of AKI could help reduce the deleterious effects of 
AKI in septic patients, such as increased illness severity and mortality [6].

The combination of vancomycin plus piperacillin-tazobactam (VPT) is one of the more frequently used broadspectrum empiric regimens for sepsis. Separately, they are two of the four most used antimicrobials in U.S. hospitals [7]. However, using VPT for more than 48 or $72 \mathrm{~h}$ increases the risk of nephrotoxicity [8-13], with possibly the greatest risk at day 5 of therapy [14]. The evidence for nephrotoxicity with brief use of VPT is less robust, with one study evaluating use of VPT versus vancomycin plus cefepime (VC) for less than $72 \mathrm{~h}$ suggesting no substantial increase in nephrotoxicity with VPT use [15].

VPT nephrotoxicity may prompt physicians to treat sepsis empirically with VC, but this substitution is far from a simple solution as cefepime is associated with other clinical effects. While patients with reduced renal function may seem ideal to receive $\mathrm{VC}$, they are at increased risk of cefepimeassociated neurotoxicity [16]. Cefepime also lacks the anaerobic organism coverage that piperacillin-tazobactam provides, which may be desired in certain clinical scenarios. A large reduction in AKI may counterbalance these disadvantages, but the extent of nephrotoxicity reduction with $\mathrm{VC}$ is not well described for initial empiric therapy in septic patients.

When nephrotoxicity from VPT is apparent in septic patients, de-escalating empiric antibiotic therapy within a 72-h period may be a feasible strategy. Empiric regimens can often be tailored based on evolving clinical information such as polymerase chain reaction testing of the nares for Methicillin resistant Staphylococcus aureus (MRSA), culture data, and other clinical testing. Whether such test findings reduce the duration of VPT use and mitigate nephrotoxicity is unclear. Further, in circumstances where septic patients can be de-escalated based on clinical data, it is uncertain whether initial empiric therapy with VPT results in a higher rate of nephrotoxicity compared to VC.

Furthermore, VPT and VC have not been adequately compared on renal recovery in patients presenting to the emergency department with sepsis-mediated AKI. While there are varying definitions of renal recovery, early recovery of renal function after AKI is associated with both improved survival and reduced long-term major adverse kidney events [17-19]. Specific to bacterial sepsis-mediated AKI, prompt and appropriate antibiotics are associated with renal recovery [18]. Thus, an antimicrobial regimen that provides appropriate coverage promptly without impeding renal recovery could confer downstream benefits.

The purpose of this study was to assess rates of AKI and early renal recovery for patients presenting to the emergency department with sepsis who are treated empirically with VPT versus VC therapy. We also evaluated other critical care outcomes associated with these two treatment strategies
- e.g., ventilator-free days, length of hospital and ICU stay, and mortality. We hypothesized that VPT would result in more nephrotoxicity than $\mathrm{VC}$, but that the two groups would not differ on other critical care outcomes.

\section{Methods}

\section{Study population and data sources}

We conducted a retrospective study of septic patients. The study was approved by our Institutional Review Board. Every patient receiving an admitting ICD-10 diagnosis of severe sepsis or septic shock after presenting to our tertiary care facility emergency department between January 12 , 2019 through December 31, 2019 was assessed for study inclusion. The hospital is in an urban setting, contains 848 beds, and includes approximately 100 intensive care unit beds.

We identified 2238 consecutive patients admitted with an ICD-10 diagnosis of severe sepsis or septic shock, two diagnoses that correspond with the most recent guidelines for the definition of sepsis [20]. Figure 1 shows the inclusion and exclusion criteria for the study. We included patients who (1) presented to the emergency department with a diagnosis of sepsis, (2) were initiated on or continued antibiotics at admission, and (3) received either VPT or VC within the first $12 \mathrm{~h}$ of presentation. Patients were excluded if (1) less than 18 years of age, (2) pregnant, (3) in end-stage renal disease (ESRD) and receiving renal replacement therapy (RRT) prior to admission, (4) transferred from another facility, (5) transferred to another facility within $72 \mathrm{~h}$ of presentation, or (6) presented after cardiac arrest. Patients were grouped in the VPT or VC arm by the receipt of each regimen in the first $12 \mathrm{~h}$ of presentation to the emergency department. Patients who received both regimens in the first $24 \mathrm{~h}$ of presentation were excluded. Most variables were extracted from the electronic medical record and validated by manual review of sample charts. When manual review found errors for antibiotics, corrections were made. These instances were often due to order discontinuation, delays in administration, or orally formulated vancomycin.

\section{Definition of variables}

Based on current guidelines [20], sepsis was defined as a modified sequential organ failure assessment (SOFA) score of 2 or greater. Septic shock was defined as vasopressor requirement to maintain mean arterial pressure $(\mathrm{MAP})>65 \mathrm{mmHg}$ and repeat lactate $>2 \mathrm{mmol} / \mathrm{L}$ after initial resuscitation. A modified SOFA score was reported using the $\mathrm{SpO} 2 / \mathrm{FiO} 2$ ratio as a substitute for the $\mathrm{PaO} 2 / \mathrm{FiO} 2$ ratio, a validated alternative [21]. The 
Fig. 1 Patient inclusion and exclusion

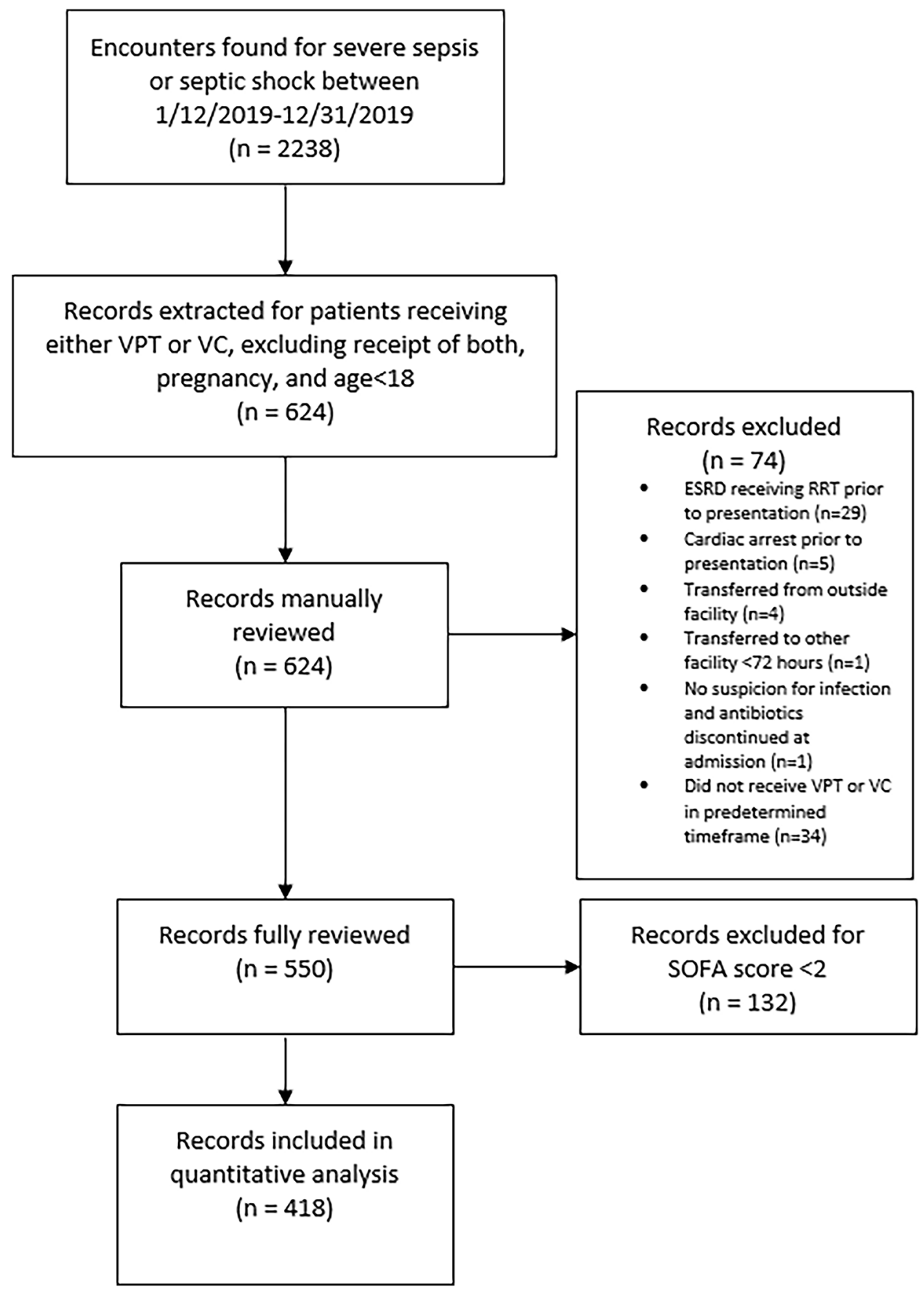

most abnormal value during each assessment period was recorded. The initial modified SOFA score was assessed in the first six hours since presentation to the emergency department, while the modified SOFA score at $72 \mathrm{~h}$ was assessed at hours 66 to 78 since presentation to the emergency department. Acute kidney injury diagnosis and staging were assessed according to Kidney Disease Improving Global Outcomes (KDIGO) criteria [22]. Urine output was omitted from the criteria due to heterogeneous documentation. Chronic Kidney Disease (CKD) was also defined according to KDIGO criteria, using an estimated glomerular filtration rate (eGFR) of $<60 \mathrm{~mL} / \mathrm{min} / 1.73 \mathrm{~m}^{2}$ calculated via the Modification of Diet in Renal Disease (MDRD) method [23]. We defined renal recovery as a $33 \%$ reduction in serum creatinine, a criterion proposed for the retrospective diagnosis of early renal recovery [24]. We assessed renal recovery at the 72 -h period as early renal recovery in this time frame is associated with reduced long-term major adverse kidney events [19]. While the $33 \%$ reduction criterion was not specifically validated for 
this timeframe, we favored this definition, which may better represent meaningful GFR changes at high creatinine values.

Secondary outcomes were evaluated either during the 72-h assessment period or during the entire hospitalization, with the assessment period specified with each variable. Intubation during hospitalization was defined as a new endotracheal intubation placed by emergency medical services or since presentation to the emergency department. Ventilator-free days were assessed for patients with intubation during hospitalization and was assessed as 28 minus days spent on mechanical ventilation since intubation. Patients who died within 28 days of intubation were quantified as 0 ventilator-free days. Vancomycin use at $72 \mathrm{~h}$ was defined as the absence of vancomycin discontinuation prior to the 72-h assessment period, regardless of dosing. Same VPT or VC regimen at $72 \mathrm{~h}$ was defined as the absence of discontinuation of both vancomycin plus piperacillin-tazobactam and vancomycin plus cefepime prior to the $72-\mathrm{h}$ assessment period, regardless of dosing.

\section{Antibiotic dosing and oversight}

Piperacillin-tazobactam dosing was according to our facility guidelines. Our standard dosing guidelines for piperacillin-tazobactam administration are an extended infusion of either $3.375 \mathrm{~g}$ or $4.5 \mathrm{~g}$ every 8 hours. Vancomycin and cefepime are dosed at the discretion of the primary provider or by referral to pharmacy for dosing recommendations. The vancomycin dosing protocol utilized trough measurements for monitoring. Antimicrobials administered in the ICU were reviewed every weekday by a clinical pharmacist rounding with the critical care team. The antimicrobial stewardship team, including an infectious disease physician and infectious disease pharmacist, prospectively audited patients receiving multiple antibiotics every weekday. Antibiotics were de-escalated or adjusted at the discretion of the primary provider or at the recommendation of the antimicrobial stewardship program. Nasal MRSA polymerase chain reaction (PCR) was routinely used as a method to de-escalate MRSA coverage in respiratory infections, as this has been shown to safely reduce the duration of vancomycin use [25, 26].

\section{Statistical analysis}

An a priori calculation was performed to determine the goal sample size for the study. Based on AKI incidences reported in earlier observational studies [8-14], at $80 \%$ power (beta $=0.20$ ) and alpha $=0.05$, a sample size of 330 with a 1:2 enrollment ratio for VC to VPT was needed to claim a $50 \%$ relative risk reduction of AKI to be statistically significant.
Continuous variables are summarized with the median and interquartile range. Categorical variables are summarized with counts and percentages. For group comparisons involving continuous variables, the Mann-Whitney Test was used since data seldomly followed a normal distribution. The chi-squared test was used for comparisons involving categorical variables. Analyses were conducted using IBM SPSS Statistics 25.0 (IBM, Armonk, NY).

\section{Results}

\section{Demographic and clinical characteristics}

After exclusions, 306 patients were identified in the VPT group, while 112 patients were identified in the VC group. Table 1 compares the demographic and clinical characteristics of the VPT and VC groups. The two groups did not significantly differ on all but two characteristics, repeat lactate and rate of pneumonia as the suspected source of infection. Both variables were higher for the VPT group.

\section{Primary outcomes}

Among 157 VPT patients with AKI at presentation, 129 were available for AKI assessment at $72 \mathrm{~h}$ while 56 of 67 $\mathrm{VC}$ patients were available (39 patients were discharged or died before $72 \mathrm{~h}$ ). Among the 129 VPT patients, $16.3 \%$ had AKI at $72 \mathrm{~h}$ compared to $8.9 \%$ of the VC patients [ $p=0.19]$. Among those without AKI at presentation, $14.2 \%$ VPT patients and $16.7 \% \mathrm{VC}$ patients had AKI at $72 \mathrm{~h}[p=0.71]$. The renal recovery rate for patients with $\mathrm{AKI}$ at presentation was $42.3 \%$ for VPT patients versus $40.3 \%$ for VC patients [ $p=0.78]$. Renal replacement therapy during hospitalization occurred in $6.2 \%$ VPT patients and $0.9 \%$ VC patients [ $p=0.024]$. [Table 2 and Fig. 2].

\section{Secondary outcomes}

Table 2 shows the results for the secondary outcomes, with continuous outcomes reported as median and interquartile range. The VPT group compared to the VC group had higher rates of intubation during hospitalization (26\% vs. $14 \%, p=0.015$ ) while the VC group had higher rates of remaining on the same regimen at $72 \mathrm{~h}(21 \%$ vs. $12 \%$, $p=0.013)$ and receiving vancomycin at $72 \mathrm{~h}(28 \%$ vs. $18 \%, p=0.030)$. Otherwise, the two groups did not differ on the secondary outcomes: change in SOFA score (VPT $=-1 \pm 3$ vs. $\mathrm{VC}=-1 \pm 3, p=0.97$ ), hospital length of stay (VPT $=7.6 \pm 7.6$ vs. $\mathrm{VC}=7.5 \pm 8.8$ days, $p=0.81$ ), ICU LOS $(\mathrm{VPT}=3.8 \pm 4.5$ vs. $\mathrm{VC}=3.1 \pm 4.0$ days, $p=0.20)$, ventilator-free days $(\mathrm{VPT}=19.3 \pm 26.0$ vs. $\mathrm{VC}=18.2 \pm 22.1$ days, $p=0.45)$, vasopressor use at 
Table 1 Demographic and clinical characteristics of 418 septic patients empirically treated with vancomycin piperacillin-tazobactam versus vancomycin cefepime

\begin{tabular}{|c|c|c|c|}
\hline Demographic and Clinical Characteristics* & $\begin{array}{l}\text { VPT } \\
N=306\end{array}$ & $\begin{array}{l}\mathrm{VC} \\
N=112\end{array}$ & $p$ \\
\hline \multicolumn{4}{|l|}{ Demographic Characteristics } \\
\hline Age-years & $67.0 \pm 20$ & $68.5 \pm 23$ & 0.85 \\
\hline Sex-female & $133(44)$ & $57(51)$ & 0.18 \\
\hline Ethnicity & & & 0.15 \\
\hline Caucasian & $233(76)$ & $76(68)$ & \\
\hline Black/African American & $68(22)$ & $35(31)$ & \\
\hline Asian & $2(1)$ & $1(1)$ & \\
\hline Two or more ethnicities & $3(1)$ & $0(0)$ & \\
\hline \multicolumn{4}{|l|}{ Clinical Characteristics } \\
\hline Body Mass Index ${ }^{a}$ & $27.2 \pm 9.2$ & $27.5 \pm 10.4$ & 0.52 \\
\hline Time to admission—-hours & $4.6 \pm 1.7$ & $4.5 \pm 1.7$ & 0.78 \\
\hline Time to first antibiotic—hours & $1.9 \pm 1.8$ & $2.2 \pm 2.2$ & 0.07 \\
\hline History of hepatic disease & $39(13)$ & $9(8)$ & 0.18 \\
\hline History of congestive heart failure & $58(19)$ & $21(19)$ & 0.96 \\
\hline \multicolumn{4}{|l|}{ Suspected source of infection } \\
\hline Pneumonia & $150(49)$ & $41(37)$ & 0.024 \\
\hline Urinary tract infection & $29(10)$ & $16(14)$ & 0.16 \\
\hline Skin and soft tissue & $28(9)$ & $11(10)$ & 0.84 \\
\hline Intra-abdominal & $14(5)$ & $6(5)$ & 0.74 \\
\hline Endocarditis/bacteremia & $12(4)$ & $2(2)$ & 0.44 \\
\hline Other & $3(1)$ & $1(1)$ & 1.00 \\
\hline Unknown & $70(23)$ & $35(31)$ & 0.08 \\
\hline Initial SOFA $\dagger$ score & $5.0 \pm 4.0$ & $4.0 \pm 4.0$ & 0.25 \\
\hline Initial mechanical ventilation & $45(15)$ & $10(9)$ & 0.12 \\
\hline Fluid volume in first $6 \mathrm{~h}-\mathrm{mL}$ & $2000 \pm 1800$ & $2000 \pm 1531$ & 0.82 \\
\hline Vasopressor within first $6 \mathrm{~h}$ & $29(10)$ & $11(10)$ & 0.92 \\
\hline Initial lactate $^{\mathrm{b}}-\mathrm{mmol} / \mathrm{L}$ & $2.70 \pm 2.90$ & $2.65 \pm 2.25$ & 0.43 \\
\hline Repeat lactate $^{c}-\mathrm{mmol} / \mathrm{L}$ & $2.30 \pm 2.30$ & $1.85 \pm 1.53$ & 0.034 \\
\hline Septic shock & $14(5)$ & $8(7)$ & 0.30 \\
\hline Creatinine prior to admission ${ }^{\mathrm{d}}-\mathrm{mg} / \mathrm{dL}$ & $0.8 \pm 0.5$ & $0.8 \pm 0.5$ & 0.31 \\
\hline Initial creatinine $-\mathrm{mg} / \mathrm{dL}$ & $1.2 \pm 1.0$ & $1.3 \pm 1.1$ & 0.25 \\
\hline Estimated GFR $\ddagger$ prior to admission $-\mathrm{mL} / \mathrm{min} / 1.73 \mathrm{~m}^{2}$ & $86 \pm 71$ & $87 \pm 77$ & 0.52 \\
\hline Initial estimated GFR $\ddagger-\mathrm{mL} / \mathrm{min} / 1.73 \mathrm{~m}^{2}$ & $58 \pm 51$ & $55 \pm 56$ & 0.18 \\
\hline Acute kidney injury at presentation & $157(51)$ & $67(60)$ & 0.12 \\
\hline Acute kidney injury stage at presentation & & & 0.07 \\
\hline No acute kidney injury & $149(49)$ & $45(40)$ & \\
\hline Stage 1 & $83(27)$ & $26(23)$ & \\
\hline Stage 2 & $40(13)$ & $25(22)$ & \\
\hline Stage 3 & 34 (11) & $16(14)$ & \\
\hline
\end{tabular}

*Continuous variables are reported as median \pm interquartile range; categorical variables are reported as count (percentage)

**Mann-Whitney Test for continuous variables; chi-squared test for categorical variables

Sample sizes: ${ }^{\mathrm{a} V P T}=253 ; \mathrm{VC}=97$

${ }^{\mathrm{b}} \mathrm{VPT}=298 ; \mathrm{VC}=110$

${ }^{c} \mathrm{VPT}=267 ; \mathrm{VC}=98$

${ }^{\mathrm{d}} \mathrm{VPT}=234 ; \mathrm{VC}=86$

${ }^{\mathrm{e}} \mathrm{VPT}=234 ; \mathrm{VC}=86$

${ }^{\dagger}$ Sequential Organ Failure Assessment

${ }^{\ddagger}$ Glomerular filtration rate 
Table 2 Outcomes of 418 septic patients empirically treated with vancomycin piperacillintazobactam versus vancomycin cefepime

\begin{tabular}{|c|c|c|c|}
\hline Outcomes* & $\begin{array}{l}\text { VPT } \\
N=306\end{array}$ & $\begin{array}{l}\mathrm{VC} \\
N=112\end{array}$ & $p$ \\
\hline \multicolumn{4}{|l|}{ Primary Outcomes } \\
\hline $\mathrm{AKI}$ at presentation with $\mathrm{AKI}$ at 72 hours $^{\mathrm{a}}$ & $21(16.3)$ & $5(8.9)$ & 0.19 \\
\hline No AKI at presentation with AKI at 72 hours $^{\mathrm{b}}$ & $18(14.2)$ & $6(16.7)$ & 0.71 \\
\hline $\mathrm{AKI}$ at presentation with renal recovery at 72 hours $^{\mathrm{c}}$ & $66(42.3)$ & $27(40.3)$ & 0.78 \\
\hline Renal replacement therapy during hospitalization & $19(6.2)$ & $1(0.9)$ & 0.024 \\
\hline \multicolumn{4}{|l|}{ Secondary Outcomes } \\
\hline \multicolumn{4}{|l|}{ Secondary Renal Outcomes } \\
\hline Change In estimated GFR $\ddagger$ from initial to 72 hours $^{\mathrm{e}}$ & $15 \pm 40$ & $15 \pm 43$ & 0.49 \\
\hline Creatinine at 72 hours $^{\mathrm{e}}$ & $0.9 \pm 0.7$ & $1.1 \pm 1.1$ & 0.55 \\
\hline Acute kidney injury stage at 72 hours $^{f}$ & & & 0.14 \\
\hline No AKI & $217(85)$ & $81(88)$ & \\
\hline Stage 1 & $24(9)$ & $9(10)$ & \\
\hline Stage 2 & $4(2)$ & $2(2)$ & \\
\hline Stage 3 & $11(4)$ & $0(0)$ & \\
\hline Time to renal replacement therapy - hours ${ }^{7}$ & $38 \pm 75$ & $74 \pm 0$ & 0.70 \\
\hline $\mathrm{RRT}$ at $72 \mathrm{~h}$ & $10(3)$ & $0(0)$ & 0.12 \\
\hline RRT discontinued $^{\mathrm{g}}$ & $6(32)$ & $0(0)$ & 1.00 \\
\hline \multicolumn{4}{|l|}{ Mechanical Ventilation } \\
\hline Intubated during hospitalization & $78(26)$ & $16(14)$ & 0.015 \\
\hline Ventilator-free days & $19.3 \pm 26.0$ & $18.2 \pm 22.1$ & 0.45 \\
\hline \multicolumn{4}{|l|}{ Antibiotic Use at 72 Hours } \\
\hline Same VPT or VC regimen at $72 \mathrm{~h}$ & $36(12)$ & $24(21)$ & 0.013 \\
\hline Vancomycin use at $72 \mathrm{~h}$ & $55(18)$ & $31(28)$ & 0.030 \\
\hline \multicolumn{4}{|l|}{ Other Secondary Outcomes } \\
\hline Change in SOFA $\dagger$ score $^{\mathrm{d}}$ & $-1 \pm 3$ & $-1 \pm 3$ & 0.97 \\
\hline Vasopressor use at $72 \mathrm{~h}$ & $11(4)$ & $3(3)$ & 0.88 \\
\hline Hospital length of stay—days & $7.6 \pm 7.6$ & $7.5 \pm 8.8$ & 0.81 \\
\hline ICU length of stay—days & $3.8 \pm 4.5$ & $3.1 \pm 4.0$ & 0.20 \\
\hline In-hospital mortality & $45(15)$ & $11(10)$ & 0.19 \\
\hline
\end{tabular}

*Continuous variables reported as median \pm interquartile range; categorical variables reported as count (percentage)

**Mann-Whitney Test for continuous variables; chi-squared test for categorical variables

***Two proportion z-test

Sample sizes: ${ }^{\mathrm{a}} \mathrm{VPT}=129 ; \mathrm{VC}=56$

${ }^{\mathrm{b}} \mathrm{VPT}=127 ; \mathrm{VC}=36$

${ }^{\mathrm{c}} \mathrm{VPT}=156 ; \mathrm{VC}=67$

${ }^{\mathrm{d}} \mathrm{VPT}=265 ; \mathrm{VC}=92$

${ }^{\mathrm{e}} \mathrm{VPT}=255 ; \mathrm{VC}=92$

${ }^{\mathrm{f}} \mathrm{VPT}=256 ; \mathrm{VC}=92$

${ }^{\mathrm{g}} \mathrm{VPT}=19 ; \mathrm{VC}=1$

${ }^{\dagger}$ Sequential Organ Failure Assessment
$72 \mathrm{~h}(\mathrm{VPT}=4 \%$ vs. $\mathrm{VC}=3 \%, p=0.88)$, change in estimated GFR from initial to $72 \mathrm{~h}(\mathrm{VPT}=15 \pm 40 \mathrm{vs}$. $\left.\mathrm{VC}=15 \pm 43 \mathrm{~mL} / \mathrm{min} / 1.73 \mathrm{~m}^{2}, p=0.49\right)$, creatinine at $72 \mathrm{~h}(\mathrm{VPT}=0.9 \pm 0.7 \mathrm{vs} . \mathrm{VC}=1.1 \pm 1.1 \mathrm{mg} / \mathrm{dL}, p=0.55)$, acute kidney injury stage at $72 \mathrm{~h}(p=0.14)$, time to renal replacement therapy (VPT $=38 \pm 75$ vs. $\mathrm{VC}=74 \pm 0 \mathrm{~h}$, $p=0.70)$, RRT at $72 \mathrm{~h}(\mathrm{VPT}=3 \%$ vs. $\mathrm{VC}=0 \%, p=0.12)$,
RRT discontinued (VPT $=32 \%$ vs. $\mathrm{VC}=0 \%, p=1.00$ ), and in-hospital mortality (VPT $=15 \%$ vs. $\mathrm{VC}=10 \%$, $p=0.19$ ). 
Fig. 2 Nephrotoxicity outcomes for patients initially treated with either VPT or VC in sepsis.

\section{Nephrotoxicity Outcomes}

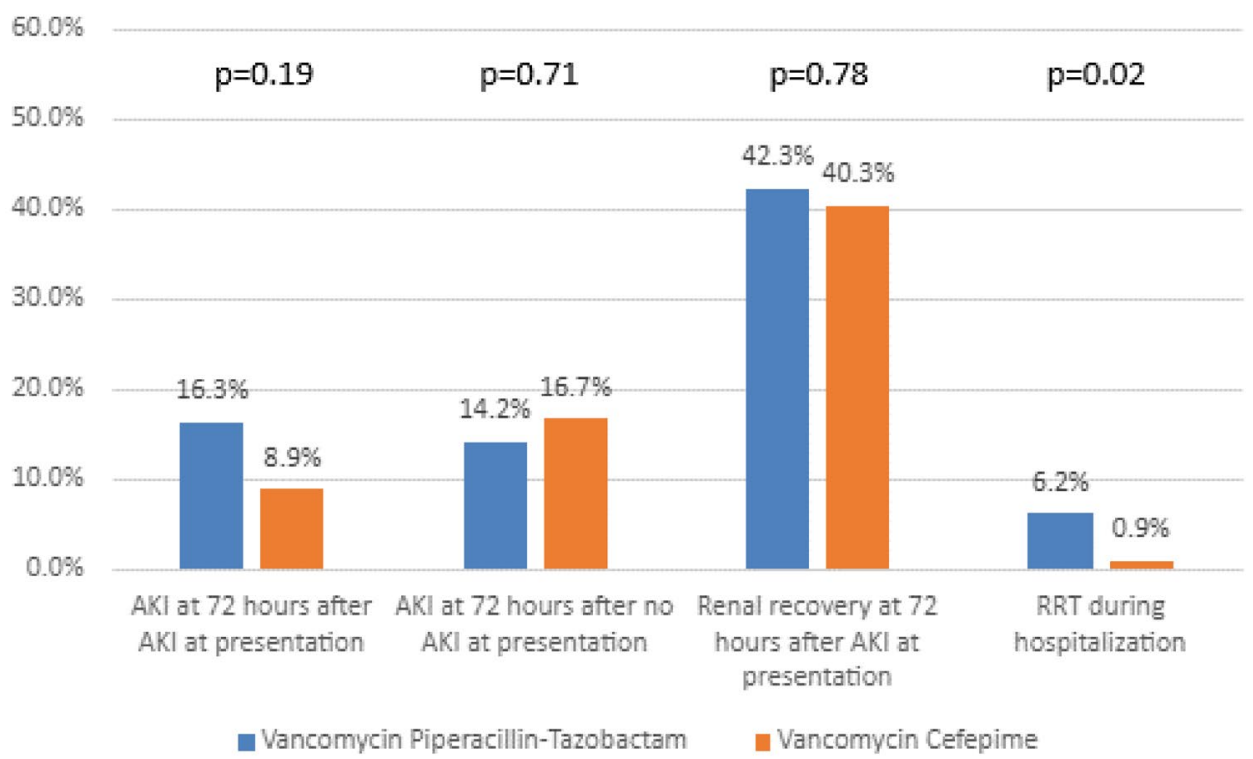

\section{Discussion}

Our study adds to the evidence on AKI risk for VPT and $\mathrm{VC}$ by evaluating patients receiving initial empiric therapy after presenting to the emergency department with sepsis defined by current guidelines. Further, we evaluated renal recovery among septic patients initially presenting with AKI.

We found no increase in AKI or decrease in renal recovery at $72 \mathrm{~h}$ with VPT therapy as compared with VC therapy. Prolonged VPT therapy has a 2- to threefold increased risk of AKI compared to VC [8-13]. In our patients, early deescalation $(<20 \%$ receiving the same initial empiric regimen at $72 \mathrm{~h}$ ) likely accounted for the absence of increased AKI risk. This finding emphasizes the importance of deescalating broad-spectrum antimicrobial therapy early, when feasible. The lack of differences in demographic and clinical characteristics before and at admission in the VPT or VC groups suggests that clinicians may have chosen a providerpreferred regimen. One notable exception to the lack of differences is the higher rate of pneumonia as the suspected source of sepsis among patients treated with VPT. This difference may indicate some preference for VPT over VC for the treatment of pneumonia.

The risk of VPT nephrotoxicity was less clear for patients with AKI at presentation. Patients who received VPT versus $\mathrm{VC}$ had an $83 \%$ relative risk increase for AKI at $72 \mathrm{~h}$ (VPT: 16.3\%; VC: $8.9 \%$ ) that was not statistically significant but may be clinically meaningful. For patients without AKI at presentation, those who received VPT versus VC had a $15 \%$ relative risk reduction for $\mathrm{AKI}$ at $72 \mathrm{~h}$ (VPT: $14.2 \%$; VC: $16.7 \%$ ), also not statistically significant.
We found an increased risk of renal replacement therapy during hospitalization for patients who received VPT (19 patients [6.2\%]) as compared with VC (1 patient $[0.9 \%])$, despite the $\mathrm{VC}$ patients demonstrating comparable AKI rates to that of VPT patients. Most patients who received RRT presented with AKI, $84 \%$ of VPT patients who received RRT and $100 \%$ of VC patients who received RRT. The findings for VPT patients-a $83 \%$ relative risk increase for AKI at $72 \mathrm{~h}$ for patients with initial AKI and more renal replacement therapy during hospitalization-may indicate that VPT nephrotoxicity is more likely among patients with preceding nephrotoxic insult, even when antimicrobial therapy is de-escalated.

Our study had several limitations that may affect its application to clinical practice. The study was conducted at a single hospital. Consequently, generalizability to other clinical settings should be done with caution. Antimicrobial stewardship at our hospital might also impact generalizability, as not all centers may review antimicrobial use for appropriateness on such a frequent basis. Since this study was a retrospective chart review investigation, data omissions occurred more frequently than in a prospective study. The clinical assessment of renal function also carries limitations. Creatinine and calculated eGFR were used as markers to determine renal function and nephrotoxicity. These surrogates are imperfect, especially in the setting of acute kidney injury of the critically ill. However, they are often used in clinical practice. Urine output was not able to be assessed due to its variable documentation. Lack of a uniform definition of renal recovery in the literature limits the assessment of this clinical outcome. Finally, the study's retrospective design precluded 
statements of causation, restricting our findings to correlational relationships.

\section{Conclusion}

Initial empiric therapy with VPT or VC for septic patients presenting to the emergency department did not differ on rates of acute kidney injury and renal recovery at $72 \mathrm{~h}$. Consequently, initial empiric therapy with VPT may not result in more nephrotoxicity than VC for septic patients, provided antimicrobial therapy is de-escalated appropriately.

Acknowledgements Douglas Hall for his work in data extraction for the study. This work was performed at the Department of Critical Care Medicine at Miami Valley Hospital, Dayton, $\mathrm{OH}$

\section{Declarations}

Conflict of interest None of the authors have conflicts of interest to report with regard to this research project. We have no financial conflicts of interest to disclose as well as no financial support to disclose.

Disclaimer The views expressed are those of the authors and do not necessarily reflect the official views of the Wright Patterson Medical Center, United States Air Force, or the Department of Defense. Mention of trade names, commercial products, or organizations does not imply endorsement by the U.S. Government.

Ethics Approval Ethical approval and informed consent were waived by the local Ethics Committee (IRB) of Wright State University in view of the retrospective nature of the study and all the procedures being performed were part of the routine care.

\section{References}

1. Rhee C, Dantes R, Epstein L et al (2017) CDC prevention epicenter program: incidence and trends of sepsis in US hospitals using clinical vs claims data, 2009-2014. JAMA 318:1241-1249

2. Rubens M, Saxena A, Ramamoorthy V et al (2020) Increasing sepsis rates in the United States: results from National Inpatient Sample, 2005 to 2014. J Intensive Care Med 35:858-868

3. Ferrer R, Martin-Loeches I, Phillips G et al (2014) Empiric antibiotic treatment reduces mortality in severe sepsis and septic shock from the first hour: results from a guideline-based performance improvement program. Crit Care Med 42:1749-1755

4. Dellinger RP, Levy MM, Rhodes A et al (2013) Surviving sepsis campaign: international guidelines for management of severe sepsis and septic shock, 2012. Crit Care Med 41:580-637

5. Poukkanen M, Vaara ST, Pettilä V et al (2013) Acute kidney injury in patients with severe sepsis in Finnish intensive care units. Acta Anaesthesiol Scand 57:863-872

6. Uchino S, Kellum JA, Bellomo R et al (2005) Acute renal failure in critically ill patients: a multinational, multicenter study. JAMA 294:813-818

7. Magill SS, O'Leary E, Ray SM et al (2020) Antimicrobial Use in US Hospitals: Comparison of Results from Emerging Infections Program Prevalence Surveys, 2015 and 2011. Clin Infect Dis 72(10): $1784-1792$
8. Navalkele B, Pogue JM, Karino S et al (2017) Risk of acute kidney injury in patients on concomitant vancomycin and piperacillintazobactam compared to those on vancomycin and cefepime. Clin Infect Dis 64:116-123

9. Rutter WC, Cox JN, Martin CA et al (2017) Nephrotoxicity during vancomycin therapy in combination with piperacillin-tazobactam or cefepime. Antimicrob Agents Chemother 61:e02089-e2116

10. Moenster RP, Linneman TW, Finnegan PM, Hand S, Thomas Z, McDonald JR (2014) Acute renal failure associated with vancomycin and $\beta$-lactams for the treatment of osteomyelitis in diabetics: piperacillin-tazobactam as compared with cefepime. Clin Microbiol Infect 20:O384-O389

11. Gomes DM, Smotherman C, Birch A et al (2014) Comparison of acute kidney injury during treatment with vancomycin in combination with piperacillin-tazobactam or cefepime. Pharmacotherapy 34:662-669

12. Peyko V, Smalley S, Cohen H (2017) Prospective comparison of acute kidney injury during treatment with the combination of piperacillin-tazobactam and vancomycin versus the combination of cefepime or meropenem and vancomycin. J Pharm Pract 30:209-213

13. Hammond DA, Smith MN, Painter JT, Meena NK, Lusardi K (2016) Comparative incidence of acute kidney injury in critically ill patients receiving vancomycin with concomitant piperacillintazobactam or cefepime: a retrospective cohort study. Pharmacotherapy 36:463-471

14. Karino S, Kaye KS, Navalkele B et al (2016) Epidemiology of acute kidney injury among patients receiving concomitant vancomycin and piperacillin-tazobactam: opportunities for antimicrobial stewardship. Antimicrob Agents Chemother 60:3743-3750

15. Schreier DJ, Kashani KB, Sakhuja A et al (2019) Incidence of acute kidney injury among critically Ill patients with brief empiric use of antipseudomonal $\beta$-lactams with vancomycin. Clin Infect Dis 68(9): 1456-1462

16. Khan A, DeMott JM, Varughese C, Hammond DA (2020) Effect of cefepime on neurotoxicity development in critically ill adults with renal dysfunction. Chest 158(1):157-163

17. Kellum JA, Sileanu FE, Bihorac A et al (2017) Recovery after acute kidney injury. Am J Respir Crit Care Med 195:784-791

18. Sood MM, Shafer LA, Ho J et al (2014) Early reversible acute kidney injury is associated with improved survival in septic shock. J Crit Care 29:711-717

19. Bhatraju PK, Zelnick LR, Chinchilli VM et al (2020) Association between early recovery of kidney function after acute kidney injury and long-term clinical outcomes. JAMA Netw Open. 3(4):e202682

20. Singer M, Deutschman CS, Seymour CW et al (2016) The third international consensus definitions for sepsis and septic shock (Sepsis-3). JAMA 315(8):801-810

21. Pandharipande PP, Shintani AK, Hagerman HE et al (2009) Derivation and validation of Spo2/Fio2 ratio to impute for Pao2/Fio2 ratio in the respiratory component of the Sequential Organ Failure Assessment score. Crit Care Med 37(4):1317-1321

22. Disease K (2012) Improving Global Outcomes (KDIGO) Acute Kidney Injury Work Group KDIGO Clinical Practice Guideline for Acute Kidney Injury. Kidney Int Suppl 2:1-138

23. Levey AS, Bosch JP, Lewis JB et al (1999) A more accurate method to estimate glomerular filtration rate from serum creatinine: a new prediction equation. Modification of Diet in Renal Disease Study Group. Ann Intern Med 130(6):461-470

24. Duff S, Murray PT (2020) Defining Early Recovery of Acute Kidney Injury. Clin J Am Soc Nephrol 15(9):1358-1360

25. Dangerfield B, Chung A, Webb B, Seville MT (2014) Predictive value of methicillin-resistant Staphylococcus aureus (MRSA) nasal swab PCR assay for MRSA pneumonia. Antimicrob Agents Chemother 58(2):859-864 
26. Dadzie P, Dietrich T, Ashurst J (2019) Impact of a Pharmacistdriven Methicillin-resistant Staphylococcus aureus Polymerase Chain Reaction Nasal Swab Protocol on the De-escalation of Empiric Vancomycin in Patients with Pneumonia in a Rural Healthcare Setting. Cureus. 11(12):e6378
Publisher's Note Springer Nature remains neutral with regard to jurisdictional claims in published maps and institutional affiliations. 